\title{
Lupus Related Longitudinal Myelitis
}

PANAGIOTIS ZOTOS, MD; JOHN POULARAS, MD, Intensive Care Unit, General State Hospital, Athens; DIMITRIOS KARAKITSOS, MD, PhD; ANDREAS KARABINIS, MD, PhD, Intensive Care Unit, General State Hospital, Athens, Greece. Address correspondence to Dr. P. Zotos, Intensive Care Unit, General State Hospital, Mesogeion 154, Athens 11523, Greece. E-mail: pzotos@endo.gr. J Rheumatol 2010;37:1776; doi:10.3899/jrheum.100282

Longitudinal myelitis is a rare form of lupus-related myelopathy with poor prognosis. Most patients experience partial response to treatment or relapse within the first year of diagnosis ${ }^{1}$. Longitudinal myelitis may be the presenting manifestation of systemic lupus erythematosus and may be associated with the presence of antiphospholipid antibodies $(\mathrm{aPL})^{2}$. We describe a case of longitudinal myelitis treated with pulse methylprednisolone and plasmapheresis.

A 28-year old woman with systemic lupus erythematosus presented with flaccid paralysis in all extremities and respiratory failure. She was intubated and admitted to the intensive care unit. Laboratory studies showed increased erythrocyte sedimentation rate, elevated double-stranded DNA, and low complement concentrations. Cerebrospinal fluid analysis was inconclusive. Brain and spinal cord T2-weighted magnetic resonance imaging (MRI) revealed edema and high signal intensities from the medullary-pontine junction to conus medullaris, the signal being more pronounced as far as the level of the fourth thoracic vertebra (Figure 1A). The findings were consistent with the diagnosis of acute longitu- dinal myelitis. She underwent treatment with pulse methylprednisolone, followed by 16 cycles of plasmapheresis. The MRI findings and neurological deficits subsided 3 weeks after admission (Figure 1B).

In our case neither aPL nor other clinical signs or symptoms of active lupus were detected. Longitudinal myelitis needs to be recognized promptly, because of its catastrophic onset and unfavorable outcome ${ }^{1,2}$. Therapeutic options include intravenous high-dose corticosteroids, plasmapheresis, intravenous immunoglobulin, and monthly intravenous pulsed cyclophosphamide.

\section{REFERENCES}

1. Tellez-Zenteno JF, Remes-Troche JM, Negrete-Pulido RO, Dávila-Maldonado L. Longitudinal myelitis associated with systemic lupus erythematosus: clinical features and magnetic resonance imaging of six cases. Lupus 2001;10:851-6.

2. Mula M, Bolamperti L, Varassi C, Mittino D, Colombi S, Stecco A, et al. SLE-related longitudinal myelitis with favorable outcome. Can J Neurol Sci 2009;36:393-7.
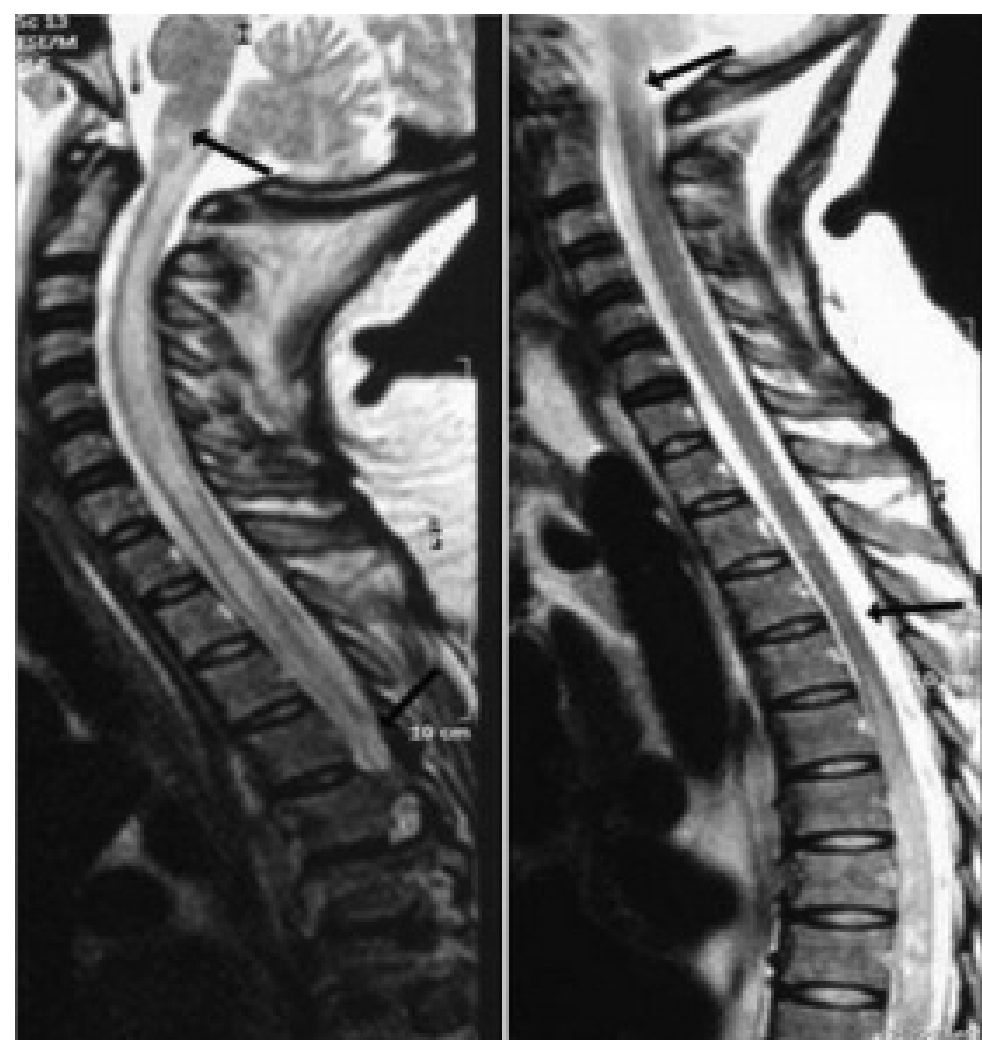

Figure 1. T2-weighted MRI of spinal cord shows high signal intensities from the medullary-pontine junction to the fourth thoracic vertebra (A, arrows). These findings subsided after therapy, 3 weeks after admission (B, arrows). 